\title{
LA INTENCIÓN ES LO QUE CUENTA. UNA REVISIÓN SOBRE LA DETECCIÓN DEL ENGAÑO EN INTENCIONES
}

\section{IT'S THE INTENTION THAT COUNTS. A REVIEW ON DECEPTION DETECTION FOCUSED ON INTENTIONS}

\author{
María Carmen Feijoo Fernández' y Lucía Halty ${ }^{2}$ \\ 'Guardia Civil. 'Universidad Pontificia Comillas
}

Durante años la investigación sobre detección del engaño ha estado guiada por las teorías clásicas que sustentaban la idea de que el mentiroso emitía indicadores conductuales que lo delataban. Dentro de las nuevas líneas de investigación, ha surgido la detección del engaño focalizado en intenciones. Haremos un repaso de los estudios sobre la detección del engaño focalizados en intenciones futuras. Explicaremos brevemente el pensamiento episódico futuro (EFT), a continuación las primeras aproximaciones en este campo a través de la comparativa entre el engaño sobre hechos pasados vs futuros. Veremos además aquellos estudios que utilizan las preguntas inesperadas en un dominio no anticipado (fase de planificación de un viaje, calidad de esa planificación y sobre el pensamiento episódico futuro), y finalizaremos por los que manejan la evidencia de manera estratégica.

Palabras clave: Detección engaño, Intenciones, Pensamiento episódico futuro (EFT).

For years the research on deception detection has been guided by classical theories that support the idea that the liar gives out behavioral indicators which betray him/her. Within the new lines of research, deception detection focused on intentions has emerged. In this paper we review the studies on deception detection focused on intentions. We briefly explain episodic future thought (EFT) and the first approaches in this field through the comparison of deception detection in past and future events. Additionally, we take a look at the studies that use unexpected questions in a non anticipated domain (trip planning phase, quality of the planning, and EFT), and we finish by discussing the ones that use evidence strategically.

Key words: Deception detection, Intentions, Episodic Future Thought (EFT).

D

urante décadas la investigación sobre la detección del engaño sentaba sus bases en el hecho de que un mentiroso podía ser detectado gracias a la emisión de claves conductuales que ponían en evidencia la falsedad de su relato. Varias han sido las teorías que intentaban sustentar y explicar esta afirmación: la hipótesis del filtraje (Ekman \& Friesen, 1969), la teoría del factor múltiple (Zuckerman, DePaulo, \& Rosenthal, 1981), la teoría autopresentacional (DePaulo, 1992; DePaulo et al., 2003) y la teoría del engaño interpersonal (Buller \& Burgoon, 1994). Dentro del ámbito forense se desarrollaron diferentes técnicas para ayudar a establecer la credibilidad de víctimas, testigos así como detectar el posible engaño en las declaraciones de los delincuentes y criminales. Un ejemplo es la SVA (Statement Validity Analysis), cuya parte central, el CBCA (Criteria Based Content Analysis; Steller \& Köhnken, 1989), ha extendido su uso a campos diferentes al inicial, a pesar

Recibido: 10 abril 2017 - Aceptado: 27 septiembre 2017 Correspondencia: María Carmen Feijoo Fernández. Cabo $1^{\circ}$ de la Guardia Civil, Unidad Fiscal y Aeroportuaria de Comandancia, Aeropuerto Adolfo Suárez Madrid Barajas, Frente Terminal 3.28042 Madrid.España.E-mail: carmenfeijoo@guardiacivil.es de las críticas efectuadas en este sentido (Köhnken, Manzanero, \& Scott, 2015). Otros ejemplos lo constituyen el control de la realidad (RM, Reality Monitoring; Johnson \& Raye, 1981), o la técnica Reid (Inbau, Reid, Buckley, \& Jayne, 2011). Esta última se enseña de manera habitual en los cursos de formación para cuerpos policiales en todo el mundo, pese a carecer de base científica (para una revisión reciente; Masip \& Herrero, 2015a).

Los resultados hallados en metaanálisis recientes han puesto de manifiesto, por un lado, la debilidad de las claves conductuales y su escasa relación con el engaño, y por otro que las claves de naturaleza verbal se relacionan en mayor medida con el engaño que las de carácter no verbal (DePaulo et al., 2003). Además, no somos buenos clasificando sinceros y mentirosos ya que rondamos el azar (Aamodt \& Custer, 2006; Bond \& Depaulo, 2008). Tampoco son mejores aquellos pertenecientes a colectivos como policías, jueces, o médicos para quienes es fundamental la detección del engaño (Bond \& DePaulo, 2006).

Todo ello hizo que primase la búsqueda de nuevos métodos para maximizar la diferencia entre mentirosos y sinceros. Por ello, surgen técnicas basadas en el incremento de la carga cognitiva, donde además, el entrevis- 
tador juega un papel activo. Un ejemplo de esta técnica lo encontramos en las entrevistas donde el entrevistador solicita el relato en orden cronológico inverso (Vrij et al., 2008). También aquellas donde se pide al entrevistado que efectúe dos tareas simultáneamente o cuando el entrevistador plantea preguntas inesperadas (Vrii et al., 2009).

Además de las técnicas concretas citadas aparecen modelos de entrevistas específicas que incorporan una herramienta para elevar la carga cognitiva del entrevistado. Las dos más punteras son, por una parte, la TRICon (Time Restricted Integrity Confirmation; Walczyk et al., 2005) en la que se solicitan respuestas rápidas, y por otra, la SUE (Strategic Use of Evidence; Hartwig, Granhag, Strömwall, \& Vrii, 2005) en la que se maneja la evidencia de modo estratégico.

Por otro lado, y a modo de crítica hacia las técnicas de detección del engaño basadas en las claves conductuales, aparece otra corriente que busca otro tipo de indicios denominados contextuales o situacionales (Park, Levine, McCornack, Morrison, \& Ferrara, 2002) (para una revisión reciente; Masip \& Herrero, 2015b). Finalmente, y como tercera línea emergen las investigaciones focalizadas en intenciones futuras, temática en la que se centra este artículo.

En primer lugar, veremos cómo surge esta nueva línea, explicaremos el concepto de pensamiento episódico futuro, y detallaremos las primeras aproximaciones que estudian características generales de las intenciones verdaderas y falsas (Vrii, Granhag, Mann, \& Leal, 2011 ; Vrii, Leal, Mann, \& Granhag, 2011). A continuación entraremos en los estudios que plantean preguntas inesperadas relacionadas con los viajes y centradas en un aspecto no anticipado (la fase de planificación de un viaje, la calidad de los planes y el pensamiento episódico futuro) (Knieps, Granhag, \& Vrii, 2013; Mac Giolla, Granhag, \& Liu-Jönsson, 2013; Sooniste, Granhag, Knieps, \& Vrii, 2013; Warmelink, Vrii, Mann, Jundi, \& Granhag, 2012). Finalizaremos por aquellos estudios que manejan la evidencia de manera estratégica (Clemens, Granhag, \& Strömwall, 2011).

\section{EL PUNTO DE PARTIDA}

Derivado de acontecimientos relativos a atentados terroristas, tanto consumados como en grado de tentativa, aparece una nueva oleada de estudios sobre el engaño focalizados en intenciones (Granhag, 2010). La capaci- dad de detectar falsas intenciones se torna fundamental en entornos tales como el aeroportuario, donde miles de pasajeros atraviesan los controles fronterizos y de seguridad. También en ámbitos en los que utilizan entrevistas abiertas o encubiertas como los servicios de inteligencia. Por ello, se hace necesario el diseño adecuado de un protocolo de entrevistas (Granhag \& Mac Giolla, 2014; Vrii \& Granhag, 2014).

Al tratarse de un campo de estudio joven, se toma la definición de la palabra intención proveniente de los estudios sobre cognición social que la describe como aquel estado mental de un individuo que precede a una acción que tendrá lugar en el futuro (Malle, Moses, \& Baldwin, 2001).

Precisamente por tratarse de un ámbito tan reciente, se considera importante resaltar varias cuestiones. En primer lugar, tal y como apunta Granhag (2010), el correcto establecimiento de las preguntas adecuadas en la investigación. Si preguntamos acerca de la intención en la comisión de un delito, hacemos referencia a la memoria prospectiva y el implementar esa intención. Si focalizamos en cómo se forman esas intenciones delictivas, ya estamos hablando de objetivos (qué), y procesos de planificación (cómo) (Szpunar \& Tulving, 2011).

Una segunda cuestión señalada por Granhag (2010) apunta a la dificultad de establecer la veracidad de una declaración en las investigaciones del ámbito forense. Al igual que ocurre con sucesos pasados: los sospechosos tienden a anticipar posibles preguntas y preparan sus historias con antelación (Hartwig, Granhag, \& Strömwall, 2007).

La tercera y última advertencia de Granhag (2010) pone el énfasis en cómo diseñar una investigación en el campo experimental. Las claves aportadas por Granhag apuntan a un diseño en el que los participantes disponen de un tiempo para planificar un evento, que para la mitad de la muestra consiste en un delito simulado y deben elaborar una historia encubierta (intención falsa), y la otra mitad una historia real (intención verdadera). A todos se les intercepta antes de que las intenciones se lleven a cabo y ambos grupos son entrevistados (Granhag, 2010). Aun cuando éste se ha convertido en el nuevo diseño desde el que se ha venido estudiando el engaño en intenciones, se hace necesario profundizar más en las características de esas intenciones.

Tal y como apuntan Schacter y colaboradores (2008), generalmente cuando las intenciones son relevantes para 
el individuo van acompañadas de un cierto grado de planificación. Además, requiere del compromiso para la puesta en marcha para alcanzar el objetivo marcado (por ejemplo, podemos pensar en matricularnos en un gimnasio cuando llegue septiembre porque en verano hemos cogido peso -deseo de ponerlo en marcha- pero llegado el momento, no lo hacemos -no existe el compromiso necesario-), es decir, no es una verdadera intención sino, tal vez, un simple deseo (Schacter et al., 2008).

Durante el proceso de planificación de una intención, el individuo utiliza su capacidad mental para pre-experimentar los sucesos que pueden ocurrir en el futuro (Szpunar, 2010). Aunque no se trata de un concepto nuevo, a esta habilidad se le asignó por primera vez el nombre de "pensamiento episódico futuro" (EFT, Episodic Future Thinking; Atance \& O'Neill, 2001), pasando a considerarse parte esencial en el proceso de generación de intenciones.

Antes de avanzar en el desarrollo del presente artículo, explicaremos el concepto de EFT y algunas de las investigaciones relacionadas con este tipo de imágenes mentales (Jeunehomme \& D'Argembeau, 2017; Lehner \& D'Argembeau, 2016). Esto facilitará la comprensión de los siguientes apartados donde desarrollaremos los estudios sobre el engaño centrados en intenciones.

\section{PENSAMIENTO EPISÓDICO FUTURO (EFT)}

El ser humano tiene la capacidad de pre-experimentar eventos en los que se ve a sí mismo en el futuro. Esta capacidad se considera esencial en el proceso de toma de decisiones, la planificación y el logro de objetivos (Suddendorf \& Corballis, 2007). En concreto, aporta flexibilidad en la elaboración de los planes orientados a alcanzar los objetivos propuestos (Schacter et al., 2008). En gran medida, esta capacidad de imaginarse a uno mismo en una escena futura recae en la habilidad de volver a recrear escenas pasadas. Estas escenas imaginadas van muy ligadas a la memoria episódica, lo que favorece la viveza y calidad de los detalles recreados (Addis, Wong, \& Schacter, 2008; Szpunar, 2010). Además, la incapacidad de recuperación de vivencias pasadas se asocia a la misma incapacidad para imaginarse a uno mismo en el futuro, y por lo tanto, imposibilita el generar imágenes (para una revisión reciente; Szpunar \& Radvansky, 2016).

Las evidencias indican que para construir estas repre- sentaciones mentales futuras, se produce un primer acceso a la memoria semántica, y a continuación, se extraen los datos de la memoria episódica que ayudan a completar la escena (D'Argembeau \& Mathy, 2011). No solo se destaca la importancia de ambos tipos de memoria, sino que cuando se trata de un objetivo de carácter personal se favorece la construcción del pensamiento episódico futuro ( $D^{\prime}$ Argembeau \& Mathy, 2011; Lehner \& D'Argembeau, 2016). Otros hallazgos muestran que la familiaridad del lugar, de las personas y de los objetos imaginados aparecen como fuertes predictores de la viveza de las imágenes mentales recreadas. La cercanía subjetiva y la convicción de que realmente la escena suceda en el futuro, incrementan la sensación de pre-experimentar esa escena imaginada (D'Argembeau \& Van der Linden, 2012).

Ulteriores estudios relacionados con la simulación de hechos futuros muestran que los detalles de una escena imaginada, la plausibilidad de que suceda y la familiaridad de las personas imaginadas, aparecen como importantes predictores en el almacenamiento en la memoria y posterior recuperación (McLelland, Devitt, Schacter, \& Addis, 2015). Una parte esencial en la memorización y recuperación a posteriori de una simulación futura, lo constituyen las personas, los lugares y las emociones (Barsics, Van der Linden, \& D'Argembeau, 2016; McLelland et al., 2015; Robin, Wynn, \& Moscovitch, 2015; Szpunar, Addis, \& Schacter, 2012), por encima de los objetos o las acciones (Jeunehomme \& D'Argembeau, 2017).

Una vez detalladas algunas de las características del EFT, a continuación entraremos ya a desglosar las diferentes aproximaciones en las investigaciones relacionadas con la detección del engaño en intenciones.

\section{PRIMERAS APROXIMACIONES A LA DETECCIÓN DEL ENGAÑO EN INTENCIONES}

Los estudios iniciales en este ámbito datan de esta misma década. En el primero llevado a cabo en un aeropuerto internacional londinense, se preguntó a los pasajeros sobre qué iban a hacer en su lugar de destino (intenciones futuras). En las respuestas se medía: la cantidad de detalles, la plausibilidad, las contradicciones y las correcciones espontáneas. Los resultados mostraron que las declaraciones de los mentirosos eran menos plausibles, tenían más contradicciones y menos correcciones espontáneas que las declaraciones de los since- 
ros. En cambio, no se hallaron diferencias en los detalles aportados por sinceros y mentirosos (Vrii et al., 201 la).

Los mismos investigadores realizaron un segundo estudio (Vrii et al., 2011b), en el que comparaban relatos verdaderos y falsos referidos a acciones pasadas y futuras. A los participantes se les asignaba una misión como agentes encubiertos (entregar un paquete a un agente situado en un edificio concreto, siguiendo una ruta específica). Al inicio de la misión, dos agentes (uno amigo y otro enemigo) interceptaban a todos los participantes. Según un código acordado previamente, al agente amigo decían la verdad y al enemigo mentían. Tras la entrega, dos nuevos agentes (uno amigo y otro enemigo) interceptaban a todos los participantes siguiendo el mismo procedimiento. En total, a cada participante se le interceptaba en cuatro ocasiones, lo que suponía decir la verdad dos veces (una sobre intenciones y otra sobre hechos pasados) y mentir en otras dos. En los relatos sobre hechos pasados, se halló que los mentirosos aportaron menos detalles y sus declaraciones fueron menos plausibles que las de los sinceros. En los relatos sobre intenciones, no se hallaron diferencias en la cantidad de detalles entre sinceros y mentirosos, si bien las declaraciones de los mentirosos fueron menos plausibles. Las grabaciones de estos relatos fueron empleadas para evaluar la precisión para la correcta clasificación de los relatos, resultando que sobre hechos pasados se alcanzó una precisión correcta de un $55 \%$ y en intenciones de un 70\% (Vrij et al., 2011 b).

\section{PREGUNTAS ESPERADAS E INESPERADAS EN UN DOMINIO INESPERADO}

Entre las técnicas innovadoras de incremento de la carga cognitiva se halla el uso de las preguntas inesperadas (Vrij et al., 2009). Al igual que ya se hiciera en estudios sobre detección del engaño focalizado en hechos pasados, esta técnica también se ha empleado para evaluar los relatos sobre intenciones futuras (Mac Giolla \& Granhag, 2015; Warmelink et al., 2012).

Una primera aproximación, la encontramos en un estudio realizado a personas que se seleccionaron entre aquellas que iban a efectuar un viaje en un futuro cercano, y otras que no tenían previsto viajar. A las que no viajaban se les pedía que preparasen una historia sobre un viaje ficticio (Warmelink et al., 2012). Se les plantearon preguntas repartidas en cuatro categorías: a) temas generales del viaje (por ejemplo, el propósito del mis- mo), b) datos considerados esenciales (descripción en detalle de un evento principal planificado para ese viaje), c) medios de transporte (en qué medio concreto se iba a desplazar), y d) planificación (qué parte del viaje resultó más fácil de planificar). Todos consideraron la categoría general de preguntas como la más previsible, siendo en esta categoría donde los que mintieron sobre sus intenciones, mencionaron más detalles. En cambio, en el resto de categorías los mentirosos citaron menos detalles (visuales y espaciales), resultando significativo en el caso de los transportes. Los autores consideraban que los resultados podrían estar motivados por la preparación que expresamente se solicitó a los mentirosos, unido a la previsible anticipación de las preguntas de la categoría general. Por ello, alertaban que se debía actuar con cautela cuando se evaluaban los detalles emitidos en los relatos, ya que la mera cuantificación podía no ser correcta (Warmelink et al., 2012).

A partir del uso de preguntas inesperadas, se abren tres líneas diferentes de investigación sobre intenciones, pero cada una centrando las preguntas en un dominio concreto: planificación, calidad de los planes y pensamiento episódico futuro (Vrij \& Granhag, 2014).

\section{Fase de planificación}

Para abordar la fase de planificación, Sooniste y colaboradores (2013) realizaron un estudio dentro del paradigma del delito simulado en el que plantearon preguntas sobre intenciones (esperadas) y otras específicas sobre la fase de planificación (inesperadas). La mitad de la muestra debía planificar una visita a un centro comercial para comprar regalos. La otra mitad debía planificar una visita al mismo lugar pero para dejar una tarjeta de memoria conteniendo material ilegal, en una estantería dentro de una tienda concreta. A todos se les interceptaba antes de llevar a cabo los planes, debiendo contar la verdad los primeros, y una historia encubierta los segundos. Aunque sinceros y mentirosos consideraron las preguntas sobre la fase de planificación como menos anticipadas (menos esperadas), los últimos lo hicieron en mayor medida. Sinceros y mentirosos percibieron como menos difíciles de responder las preguntas sobre intenciones, y por lo tanto, como más anticipadas que las relativas a la fase de planificación. Las respuestas de sinceros y mentirosos a preguntas sobre las intenciones resultaron igual de detalladas, coincidiendo con resultados hallados en investigaciones previas (Vrii et al., 
2011 a; Vrii et al., 2011 b). Las respuestas de los sinceros a las preguntas no anticipadas fueron más largas que las de los mentirosos, pero no hubo diferencias entre sinceros y mentirosos al responder a preguntas anticipadas (Sooniste et al., 2013).

Similares resultados se encontraron en dos estudios posteriores en los que se dividía a los participantes en grupos. Por parte de Sooniste y colaboradores (2016), se diseñó un experimento en el que una muestra se dividía en grupos de dos y cuatro personas. A todos les facilitaron planos de un gran centro comercial así como diversas páginas web donde podían consultar detalles. La tarea lícita consistía en planificar una cena típica sueca para lo que debían efectuar una compra en el centro comercial. Los mentirosos debían recoger cuatro objetos situados en cuatro tiendas diferentes, por lo que necesitaban además, una historia encubierta. Antes de realizar las misiones, a todos se les interceptaba para interrogarles por separado sobre las intenciones y la planificación. Los cuartetos se dividían en grupos de dos personas cada uno, y primero se interrogaba a un grupo mientras el otro esperaba, y viceversa. Las díadas se dividían, y a cada participante se le interrogaba por separado. Los resultados mostraron que los grupos de sinceros eran más consistentes en sus respuestas que los mentirosos ante las preguntas inesperadas, pero en las respuestas a preguntas esperadas no hubo diferencias entre sinceros y mentirosos. Las respuestas a las preguntas sobre la fase de planificación y sobre intenciones fueron menos detalladas en el caso de los mentirosos (Sooniste, Granhag, Strömwall, \& Vrii, 2016).

Con el mismo diseño, Mac Giolla y Granhag (2015) plantearon un estudio en el que dividieron a la muestra en grupos de tres, efectuando además una comparativa entre entrevista única y repetida ( 3 veces sucesivas y mínimo tiempo entre ellas). Los resultados mostraron que los grupos de sinceros daban respuestas más largas y detalladas que los mentirosos, tanto para las preguntas sobre intenciones como sobre planificación. Afirmaron los autores que las entrevistas repetidas no aportaban beneficios ya que no observaron diferencias en el grado de consistencia de las declaraciones intragrupo, ni en la longitud de las mismas (Mac Giolla \& Granhag, 2015).

\section{Calidad de los planes}

Aunque en las intenciones se asume un cierto grado de planificación tanto para las verdaderas como las falsas, se espera que estos planes sean diferentes cualitativamente en su construcción (Sooniste, Granhag, Strömwall, \& Vrii, 2015). Esta segunda aproximación, pone su foco en la calidad de los planes, asumiendo que los relatos verdaderos estarán caracterizados por marcadores indicativos de una buena planificación. Un ejemplo de marcador sería el haber anticipado alguna posible complicación para la tarea (si disponen de diez minutos para ir en coche de un sitio a otro, el tener en cuenta un posible atasco). También se considera un marcador la gestión efectiva del tiempo (si disponen de diez minutos y deben realizar varias tareas, dividirse y asignar a cada persona una diferente).

En un diseño similar, Mac Giolla y colaboradores (2103) realizaron un estudio con una muestra a la que distribuyeron en grupos de tres. Asignaron la planificación de una tarea neutral a sinceros y de un delito simulado a los mentirosos, interceptando a todos ellos antes de efectuarla para interrogarles. Hallaron resultados en la línea descrita, ya que los sinceros mencionaron en más ocasiones los marcadores asignados a la buena planificación: la intención de dividirse al llegar al centro comercial y el haber anticipado algún posible contratiempo. No obstante, los autores advirtieron que estas conductas específicas iban muy ligadas al contexto de esta investigación, por lo que sus resultados no era posible generalizarlos a otras situaciones (Mac Giolla et al., 2013).

Resultados análogos encontraron Granhag y colaboradores (2016), que pusieron a prueba la consistencia en entrevistas repetidas tres veces con intervalos de diez minutos. Plantearon preguntas tanto sobre las intenciones como la planificación. Se examinaban marcadores de una buena planificación como por ejemplo, la anticipación de posibles complicaciones y el disponer de planes alternativos. Los resultados mostraron que sinceros y mentirosos percibieron las preguntas relacionadas con la fase de planificación como más inesperadas y más difíciles de responder que las relacionadas con las intenciones. Si bien, los mentirosos percibieron mayor dificultad en responder a las preguntas sobre intenciones, contrario a lo esperado por los autores. La consistencia en las entrevistas resultó similar aunque las respuestas a preguntas sobre la planificación fueron menos consistentes que las relativas a intenciones (Granhag, Mac Giolla, Sooniste, Strömwall, \& Liu-Jonsson, 2016). 


\section{Pensamiento Episódico Futuro}

Como tercera y última línea focalizada en un dominio inesperado, nos encontramos la que aborda el pensamiento episódico futuro (Atance \& O'Neill, 2001). Esta habilidad de imaginarse a uno mismo en el futuro se caracteriza por presentar imágenes mentales de gran viveza sensitivo - perceptual. La recreación de estas imágenes tiene lugar durante la fase de planificación de las intenciones verdaderas, y por ello, son parte esencial de las mismas (Szpunar, 2010). Se asume que aquellas personas con intenciones verdaderas tenderán a planificarlas de un modo más detallado y recurrirán en mayor medida a EFT's. Por el contrario, los que elaboran una historia encubierta evocarán imágenes mentales en menor medida y las características diferirán de las relativas a intenciones reales (Granhag \& Knieps, 2011).

Con base en el nuevo diseño de investigación, Granhag y Knieps (2011) realizaron el primer estudio sobre estas imágenes mentales relacionadas con acciones futuras. Durante la entrevista les plantearon varias preguntas relacionadas con las imágenes mentales imaginadas: a) en qué medida las habían evocado, b) efectuar una descripción de esa imagen lo más detallada posible, y c) cualquier otra cuestión que quisiesen añadir. Los resultados mostraron que los que relataban intenciones verdaderas afirmaron evocar las imágenes en mayor medida (97\%) que los mentirosos (66\%). Además, encontraron diferencias cualitativas, caracterizándose los relatos falsos por una menor riqueza en las descripciones (menor número de palabras empleadas) (Granhag \& Knieps, 2011 ). Por otro lado, a los participantes se les solicitaba que cumplimentasen un cuestionario en el que entre otras, se incluyeron preguntas del $\left(M C Q\right.$, Memory $\mathrm{Ca}^{-}$ racteristics Questionnaire; Johnson, Foley, Suengas, \& Raye, 1988). Se les preguntaba en qué grado habían experimentado determinados detalles en la imagen mental evocada (sensoriales de tipo auditivo, visual o sabor/olor, espaciales del tipo situación de una persona u objeto, y temporales). Tan solo encontraron diferencias en los detalles de tipo temporal, y espacial relacionados con la situación de objetos, que los sinceros plasmaron en mayor medida (Granhag \& Knieps, 2011).

Posteriormente, Knieps, Granhag y Vrii (2013) Ilevaron a cabo un estudio en el que trataron de replicar los resultados obtenidos por Granhag y Knieps (2011). También querían poner a prueba la consistencia de las descripciones en las EFT's en sinceros y mentirosos, en- trevistando dos veces en el intervalo de una semana. Analizaron los datos de las entrevistas transcritos y codificados (medida objetiva), y los correspondientes a un cuestionario cumplimentado por los participantes tras la entrevista (medida subjetiva). Los resultados de las entrevistas mostraron que los sinceros declararon haber evocado en mayor medida $\mathrm{EFT}^{\prime}$ 's (93\%) que los mentirosos (71\%), lo que mostraba el vínculo existente entre la elaboración de las intenciones y la activación de las imágenes, replicando por lo tanto el trabajo de Granhag y Knieps (2011). No encontraron diferencias entre sinceros y mentirosos en el número de palabras empleadas. En las medidas subjetivas (cuestionarios) y objetivas (transcripciones), no se hallaron claras diferencias en los detalles (sensoriales, espaciales y temporales) (Knieps et al., 2013).

En un estudio parecido, Knieps y Granhag (2013), volvieron a analizar en qué medida los participantes evocaban los EFT's, así como diferencias en los detalles utilizados en la descripción de las imágenes. Entrevistaron a los participantes dos veces el mismo día. Los resultados mostraron que los sinceros en mayor medida que los mentirosos evocaban los EFT's en la fase de planificación: $96.7 \%$ y $100 \%$ en los sinceros (primera y segunda entrevista respectivamente), frente al $76.7 \%$ y el $83.3 \%$ en los mentirosos. En línea a lo hallado por (Knieps et al., 2013), no se encontraron diferencias entre sinceros y mentirosos en los detalles analizados ni objetiva (transcripciones), ni subjetivamente (respuestas al cuestionario post-entrevista) (Knieps \& Granhag, 2013).

\section{USO ESTRATÉGICO DE LA EVIDENCIA EN INTENCIONES}

Ante la necesidad de establecer la credibilidad y detectar el engaño en contextos forenses, aparecieron diversos protocolos de entrevista. Entre ellos, María Hartwig diseñó la técnica conocida como SUE que a diferencia de otras, necesita que el entrevistador tenga una evidencia que maneja de modo estratégico durante la entrevista (Hartwig et al., 2005). La asunción principal de esta técnica radica en los diferentes estados mentales con los que sinceros y mentirosos afrontan los interrogatorios.

Empleando esta metodología, Clemens y colaboradores (2011), la pusieron a prueba para detectar falsas intenciones. Se planteaba a los participantes la preparación de una tarea que en esta ocasión comprendía además una serie de requisitos que debían llevar a cabo, lo que 
dejaba rastro a modo de evidencia (rastreo en una web, huellas en un sobre y recogida de una tarjeta de memoria). Aunque tanto sinceros como mentirosos realizaban todas estas tareas, para cada grupo tenía una finalidad distinta. Los mentirosos debían contar una historia encubierta convincente para no desvelar sus verdaderas intenciones. Se interceptó a todos antes de realizar la tarea final encomendada, $y$ todos pasaron por tres tipos de entrevista. En la primera entrevista se les confrontaba con las evidencias existentes al inicio. En las dos entrevistas siguientes, las evidencias se empleaban de modo estratégico y no se presentaban hasta el final: SUE 1: a) relato libre/preguntas específicas sobre la planificación, b) relato libre/preguntas específicas sobre intenciones; SUE 2, orden inverso. En las entrevistas que usaron las evidencias de un modo estratégico (SUE 1 y SUE 2), los resultados mostraron que las declaraciones de los mentirosos eran más inconsistentes tanto en intenciones como sobre la planificación de la tarea (en relato libre y en preguntas específicas). No se encontraron diferencias cuando la evidencia era expuesta al inicio de la entrevista. Concluyeron los autores que las entrevistas en modalidad SUE se tornaban buenas para la obtención de indicios de engaño cuando se evaluaban las intenciones (Clemens et al., 2011), al igual que se había hallado en el caso de hechos pasados (Hartwig et al., 2005).

A lo largo de este artículo hemos desarrollado los inicios de nuevas líneas de investigación en el ámbito de la detección del engaño: mentir sobre intenciones. Comenzamos exponiendo la problemática al tratarse de un campo novedoso, así como las primeras aproximaciones. Seguidamente especificamos las tres líneas principales de estudio: preguntas inesperadas vs anticipadas, sobre un dominio inesperado (planificación, calidad de los planes y EFT's), y finalizamos exponiendo las que emplean el uso estratégico de la evidencia.

\section{CONCLUSIONES}

El interés que siempre ha suscitado la detección del engaño, unido a los pobres resultados obtenidos en la clasificación de sinceros y mentirosos desde la perspectiva clásica, han guiado la constante búsqueda de nuevos métodos de detección. No obstante, la actualidad ha sido la que ha provocado la aparición de una nueva línea de estudio cuyo foco de interés se centra en hechos futuros.

Aunque ha sido en el área de la psicología forense donde surge esta nueva línea, podemos decir que son muchas las posibles áreas donde el psicólogo debe valorar si las intenciones son verdaderas o falsas. A modo de ejemplo: el psicólogo clínico debe detectar la posible ocultación de una idea suicida o evaluar la intención real de adhesión a un tratamiento; en el ámbito del trabajo y organizaciones, durante las entrevistas previas a una contratación, el psicólogo debe sondear las intenciones reales de un posible candidato; y el psicólogo educativo ha de detectar posibles intenciones de acoso antes de que se materialicen.

Por todo lo expuesto, podemos concluir que los resultados que vayan surgiendo en este nuevo campo aportarán herramientas clave que resultarán útiles en múltiples áreas de la psicología.

\section{CONFLICTO DE INTERESES}

No existe conflicto de intereses.

\section{REFERENCIAS}

Aamodt, M. G., \& Custer, H. (2006). Who can best catch a liar?: A meta-analysis of individual differences in detecting deception. Forensic Examiner, 15(1), 6.

Addis, D. R., Wong, A. T., \& Schacter, D. L. (2008). Age-related changes in the episodic simulation of future events. Psychological Science, 19(1), 33-41. doi:10.1111/j.1467-9280.2008.02043.x

Atance, C. M., \& O'Neill, D. K. (2001). Episodic future thinking. Trends in Cognitive Sciences, 5(12), 533-539. doi://dx.doi.org/10.1016/S13646613(00)01804-0

Barsics, C., Van der Linden, M., \& D'Argembeau, A. (2016). Frequency, characteristics, and perceived functions of emotional future thinking in daily life. The Quarterly Journal of Experimental Psychology, 69(2), 217-233. doi://dx.doi.org/10.1080/17470218.2015.1051560

Bond, C. F., \& DePaulo, B. M. (2006). Accuracy of deception judgments. Personality and Social Psychology Review, 10(3), 214-234. doi:10.1207/s15327957pspr1003_2

Bond, C. F., \& Depaulo, B. M. (2008). Individual differences in judging deception: Accuracy and bias. Psychological Bulletin, 134(4), 477-92. doi://psycnet.apa.org/doi/10.1037/0033-2909.134.4.477

Buller, D. B., \& Burgoon, J. K. (1994). Deception: Strategic and nonstrategic communication. En J. A. Daly \& J. M. Wiemann (Eds.), LEA's communication series. Strategic interpersonal communication (pp. 191-223). Hillsdale, NJ: Lawrence Erlbaum

Clemens, F., Granhag, P. A., \& Strömwall, L. A. (2011). 
Eliciting cues to false intent: A new application of strategic interviewing. Law and Human Behavior, 35(6), 512-522. doi:10.1007/s10979-010-9258-9

D'Argembeau, A., \& Van der Linden, M. (2012). Predicting the phenomenology of episodic future thoughts. Consciousness and Cognition, 21(3), 1198-1206. doi://dx.doi.org/10.1016/j.concog.2012.05.004

D'Argembeau, A., \& Mathy, A. (2011). Tracking the construction of episodic future thoughts. Journal of Experimental Psychology: General, 140(2), 258. doi://dx.doi.org/10.1037/a0022581

DePaulo, B. M. (1992). Nonverbal behavior and selfpresentation. Psychological Bulletin, 111 1(2), 203-243. doi:10.1037/0033-2909.111.2.203

DePaulo, B. M., Lindsay, J. J., Malone, B. E., Muhlenbruck, L., Charlton, K., \& Cooper, H. (2003). Cues to deception. Psychological Bulletin, 129(1), 74. doi://psycnet.apa.org/doi/10.1037/00332909.129.1.74

Ekman, P., \& Friesen, W. V. (1969). Nonverbal leakage and clues to deception. Psychiatry, 32(1), 88-106. doi:10.1080/00332747.1969.11023575

Granhag, P. A., \& Knieps, M. (2011). Episodic future thought: Illuminating the trademarks of forming true and false intentions. Applied Cognitive Psychology, 25(2), 274-280. doi:10.1002/acp. 1674

Granhag, P. A. (2010). On the psycho-legal study of true and false intentions: Dangerous waters and some stepping stones. The Open Criminology Journal, 3, 37-43. doi://dx.doi.org/10.2174/1874917801003010037

Granhag, P. A., \& Mac Giolla, E. (2014). Preventing future crimes. European Psychologist, 19(3), 195-206. doi://dx.doi.org/10.1027/1016-9040/a000202

Granhag, P. A., Mac Giolla, E., Sooniste, T., Strömwall, L., \& Liu-Jonsson, M. (2016). Discriminating between statements of true and false intent: The impact of repeated interviews and strategic questioning. Journal of Applied Security Research, 11(1), 1-17. doi://dx.doi.org/10.1080/19361610.2016.1104230

Hartwig, M., Granhag, A., \& Strömwall, L. A. (2007). Guilty and innocent suspects' strategies during police interrogations. Psychology, Crime \& Law, 13(2), 213227. doi:10.1080/10683160600750264

Hartwig, M., Granhag, P. A., Strömwall, L. A., \& Vrij, A. (2005). Detecting deception via strategic disclosure of evidence. Law and Human Behavior, 29(4), 469. doi:10.1007/s10979-005-5521-x
Inbau, F. E., Reid, J. E., Buckley, J. P., \& Jayne, B. C. (2011). Criminal interrogation and confessions. Jones \& Bartlett Publishers.

Jeunehomme, O., \& D'Argembeau, A. (2017). Accessibility and characteristics of memories of the future. Memory, 25, 666-676. doi://dx.doi.org/ 10.1080/09658211.2016.1205096

Johnson, M. K., Foley, M. A., Suengas, A. G., \& Raye, C. L. (1988). Phenomenal characteristics of memories for perceived and imagined autobiographical events. Journal of Experimental Psychology: General, 117(4), 371.

Johnson, M. K., \& Raye, C. L. (1981). Reality monitoring. Psychological Review, 88(1), 67. doi://psycnet.apa.org/doi/10.1037/0033-295X.88.1.67

Knieps, M., Granhag, P. A., \& Vrii, A. (2013). Back to the future: Asking about mental images to discriminate between true and false intentions. The Journal of Psychology, 147(6), 619-640. doi://dx.doi.org/10.1080/00223980.2012.728542

Knieps, M., \& Granhag, P. (2013). Repeated visits to the future: Asking about mental images to discriminate between true and false intentions. International Journal of Advances in Psychology, 2(2), 93-102.

Köhnken, G., Manzanero, A. L., \& Scott, M. T. (2015). Análisis de la validez de las declaraciones: Mitos y limitaciones. Anuario De Psicología Jurdica, 25(1), 1319. doi://dx.doi.org/10.1016/i.api.2015.01.004

Lehner, E., \& D'Argembeau, A. (2016). The role of personal goals in autonoetic experience when imagining future events. Consciousness and Cognition, 42, 267-276. doi://dx.doi.org/10.1016/i.concog.2016.04.002

Mac Giolla, E., \& Granhag, P. A. (2015). Detecting false intent amongst small cells of suspects: Single versus repeated interviews. Journal of Investigative Psychology and Offender Profiling, 12(2), 142-157. doi:10.1002/iip.1419

Mac Giolla, E., Granhag, P. A., \& Liu-Jönsson, M. (2013). Markers of good planning behavior as a cue for separating true and false intent. PsyCh Journal, 2(3), 183-189. doi:10.1002/pchi.36

Malle, B. F., Moses, L. J., \& Baldwin, D. A. (2001). Intentions and intentionality: Foundations of social cognition. Cambridge, MA: MIT press.

Masip, J., \& Herrero, C. (2015a). Nuevas aproximaciones en detección de mentiras I. Antecedentes y marco teórico. Papeles del Psicólogo, 36(2), 83-95.

Masip, J., \& Herrero, C. (2015b). Nuevas aproximacio- 
nes en detección de mentiras II: Estrategias activas de entrevista e información contextual. Papeles del Psicólogo, 36(2), 96-108.

McLelland, V. C., Devitt, A. L., Schacter, D. L., \& Addis, D. R. (2015). Making the future memorable: The phenomenology of remembered future events. Memory, 23(8), 1255-1263. doi://dx.doi.org/10.1080/09658211.2014.972960

Park, H. S., Levine, T., McCornack, S., Morrison, K., \& Ferrara, M. (2002). How people really detect lies. Communication Monographs, 69(2), 144-157. doi://dx.doi.org/10.1080/714041710

Robin, J., Wynn, J., \& Moscovitch, M. (2015). The spatial scaffold: The effects of spatial context on memory for events. Journal of Experimental Psychology: Learning, Memory and Cognition, 42(2), 308. doi://dx.doi.org/10.1037/x/m0000167

Schacter, D. L., Addis, D. R., \& Buckner, R. L. (2008). Episodic simulation of future events. Annals of the New York Academy of Sciences, 1124(1), 39-60. doi:10.1196/annals. 1440.001

Sooniste, T., Granhag, P. A., Knieps, M., \& Vrii, A. (2013). True and false intentions: Asking about the past to detect lies about the future. Psychology, Crime \& Law, 19(8), 673-685. doi://dx.doi.org/10.1080/1068316X.2013.793333

Sooniste, T., Granhag, P. A., Strömwall, L. A., \& Vrii, A. (2015). Statements about true and false intentions: Using the cognitive interview to magnify the differences. Scandinavian Journal of Psychology, 56(4), 371378. doi:10.1111/sjop. 12216

Sooniste, T., Granhag, P. A., Strömwall, L. A., \& Vrii, A. (2016). Discriminating between true and false intent among small cells of suspects. Legal and Criminological Psychology, 21(2), 344-357. doi: $10.1111 /$ lcrp. 12063

Steller, M., \& Köhnken, G. (1989). Criteria-based statement analysis. In D.C. Raskin (ED), Psychological Methods in Criminal Investigation and Evidence, 20, 217-245.

Suddendorf, T., \& Corballis, M. C. (2007). The evolution of foresight: What is mental time travel, and is it unique to humans? Behavioral and Brain Sciences, 30(03), 299-313. doi://dx.doi.org/10.1017/S0140525X07001975

Szpunar, K. K. (2010). Episodic future thought an emerging concept. Perspectives on Psychological Science, 5(2), 142162. doi://doi.org/10.1177/1745691610362350
Szpunar, K. K., Addis, D. R., \& Schacter, D. L. (2012). Memory for emotional simulations remembering a rosy future. Psychological Science, 23(1), 24-29. doi://doi.org/10.1177/0956797611422237

Szpunar, K. K., \& Radvansky, G. A. (2016). Cognitive approaches to the study of episodic future thinking. The Quarterly Journal of Experimental Psychology, 69(2), 209-216. doi://dx.doi.org/10.1080/17470218.2015.1095213

Szpunar, K. K., \& Tulving, E. (2011). Varieties of future experience. In M. In Bar (Ed.), Predictions in the brain: Using our past to generate a future (pp. 3-12). New York: Oxford University Press.

Vrii, A., Granhag, P. A., Mann, S., \& Leal, S. (201 1a). Lying about flying: The first experiment to detect false intent. Psychology, Crime \& Law, 17(7), 611-620. doi://dx.doi.org/10.1080/10683160903418213

Vrii, A., \& Granhag, P. A. (2014). Eliciting information and detecting lies in intelligence interviewing: An overview of recent research. Applied Cognitive Psychology, 28(6), 936-944. doi:10.1002/acp.3071

Vrii, A., Leal, S., Granhag, P. A., Mann, S., Fisher, R. P., Hillman, J., \& Sperry, K. (2009). Outsmarting the liars: The benefit of asking unanticipated questions. Law and Human Behavior, 33(2), 159-166. doi:10.1007/s10979-008-9143-y

Vrii, A., Leal, S., Mann, S. A., \& Granhag, P. A. $(2011 \mathrm{~b})$. A comparison between lying about intentions and past activities: Verbal cues and detection accuracy. Applied Cognitive Psychology, 25(2), 212-218. doi:10.1002/acp. 1665

Vrii, A., Mann, S. A., Fisher, R. P., Leal, S., Milne, R., \& Bull, R. (2008). Increasing cognitive load to facilitate lie detection: The benefit of recalling an event in reverse order. Law and Human Behavior, 32(3), 253-265. doi:10.1007/s10979-007-9103-y

Walczyk, J. J., Schwartz, J. P., Clifton, R., Adams, B., Wei, M., \& Zha, P. (2005). Lying person to person about life events: A cognitive framework for lie detection. Personnel Psychology, 58(1), 141-170.

Warmelink, L., Vrii, A., Mann, S., Jundi, S., \& Granhag, P. A. (2012). The effect of question expectedness and experience on lying about intentions. Acta Psychologica, 141(2), 178183. doi://dx.doi.org/10.1016\%2Fi.actpsy.2012.07.011

Zuckerman, M., DePaulo, B. M., \& Rosenthal, R. (1981). Verbal and nonverbal communication of deception. Advances in Experimental Social Psychology, 14(1), 59. doi://dx.doi.org/10.1016/S0065-2601(08)60369-X 